\title{
Acute presentation of dementia with Lewy bodies
}

\author{
Authors: Olugbenro Akintade ${ }^{A}$ and Floyd Pierres ${ }^{B}$
}

Acute presentations for dementia, particularly dementia with Lewy bodies (DLB), are rare and can pose diagnostic challenges.

We present a case of a 75-year-old woman who was previously fit, well and independent in all activities of daily living. She had no history of psychiatric, cognitive or memory problems. She presented with 2 weeks of sudden onset confusion, paranoia, dizziness and reduced oral intake. Thorough investigations for causes of delirium including blood tests, cerebrospinal fluid analysis obtained via lumbar puncture, electroencephalography, computed tomography, and magnetic resonance imaging were within normal limits. Further neurological examination demonstrated she had subtle Parkinsonian signs (cogwheel rigidity, bradykinesia) and was hypersensitive to small doses of antipsychotic (haloperidol and risperidone). A positive dopamine transporter scan was done confirming a diagnosis of an acute presentation of DLB. She has been commenced on a cholinesterase inhibitor (rivastigmine) and is presently settled in care.

KEYWORDS: Dementia, Lewy bodies, delirium, confusion

\section{Case presentation}

A 75-year-old woman presented with a 2-week history of confusion, paranoia, dizziness and reduced oral intake. She and her next of kin (son) did not report any head injury, fever, cough, chest pain, dysuria, urinary frequency, abdominal discomfort or weight loss.

Her past medical history was of benign endometrial polypectomy and hiatus hernia only.

On examination she was afebrile with a blood pressure of $181 / 98 \mathrm{mmHg}$ and a heart rate of $118 \mathrm{bpm}$. Peripheral saturations were $97 \%$ on room air. There was no significant focal neurology, cerebellar dysfunction or extrapyramidal signs noted at this stage. Her heart sounds, chest and abdomen were normal. She had some bilateral leg swelling secondary to venous insufficiency.

A mental state examination showed she was well-kempt and appeared in good mood but was abrupt in some answers and at times suspicious and guarded. There was no evidence of visual or

Authors: ${ }^{\text {A }}$ consultant physician and geriatrician, Peterborough City Hospital, Peterborough, UK; ${ }^{B}$ core medical trainee, Peterborough City Hospital, Peterborough, UK auditory hallucination, thought broadcasting, thought insertion or delusions. Her abbreviated mental state score (AMT-4 and AMT-10) were $2 / 4$ and 5/10, respectively. She was disorientated in time and place.

Her electrocardiogram showed sinus tachycardia. Her chest radiogram showed a large hiatus hernia. Her urine dip was clear.

Her bloods tests were normal including a full blood count, electrolytes, urea, calcium, magnesium, liver function, ferritin, endocrine panel, B12, folate, antinuclear antibodies, immunoglobulins, voltage gated potassium channel antibodies (include dsDNA, Sm/RNP, Ro52, Ro60, La (SS-B), Scl-70, centromere, Mi-2, Ku, Th/To, RNA Pol III, Pm-Scl and PCNA, Jo-1 and ribosomal-P, anti-neutrophil cytoplasmic immunoglobulin $G$ (IgG) antibodies screen, glutamic acid decarboxylase antibodies, anti-nuclear IgG antibodies, paraneoplastic IgG antibody screen).

Her cerebrospinal fluid analysis was grossly normal, protein $0.35 \mathrm{~g} / \mathrm{L}(0.15-0.45)$, glucose $4.1 \mathrm{mmol} / \mathrm{L}(2.2-4.0)$ and was negative for oligoclonal bands, $\mathrm{N}$-methyl-D-aspartate receptor antibodies and voltage gate potassium channel antibodies.

Her computed tomography (CT) head showed periventricular low attenuation in keeping with small vessel disease and a suspected established lacunar infarct within the anterior limb of the left internal capsule bordering on the head of the caudate. There was medial temporal lobe volume preservation.

Her magnetic resonance imaging (MRI) head showed marked involutional changes compatible with her age. There was irregular T2 white matter hyper intensity consistent with moderate microvascular ischaemia. There was however no acute lesion on diffusion-weighted imaging (DWI).

Her electroencephalography (EEG) showed an asymmetrical pattern, characterised by excess of diffuse and at times rhythmical theta activity at $4.5-7 \mathrm{~Hz}$ (up to $71 \mathrm{uV}$ ). There was occasional irregular theta components noted over the temporal region (left more than right) up to $105 \mathrm{uV}$, sporadically intermixed with underlying delta components at $1-2 \mathrm{~Hz}$ (up to $80 \mathrm{uV}$ ) over the temporal regions. Posterior dominant rhythm reactive to eye opening was not evident.

Her dopamine transporter scan (DaTSCAN) showed significantly reduced uptake of ioflupane in the right striatum (Fig 1).

\section{Differential diagnosis}

The initial diagnosis was unclear. The patient was reviewed by numerous medical teams; including acute medicine, geriatrics, neurology and psychiatry. A wide differential was established including acute delirium, new onset dementia, autoimmune encephalitis and psychosis. 


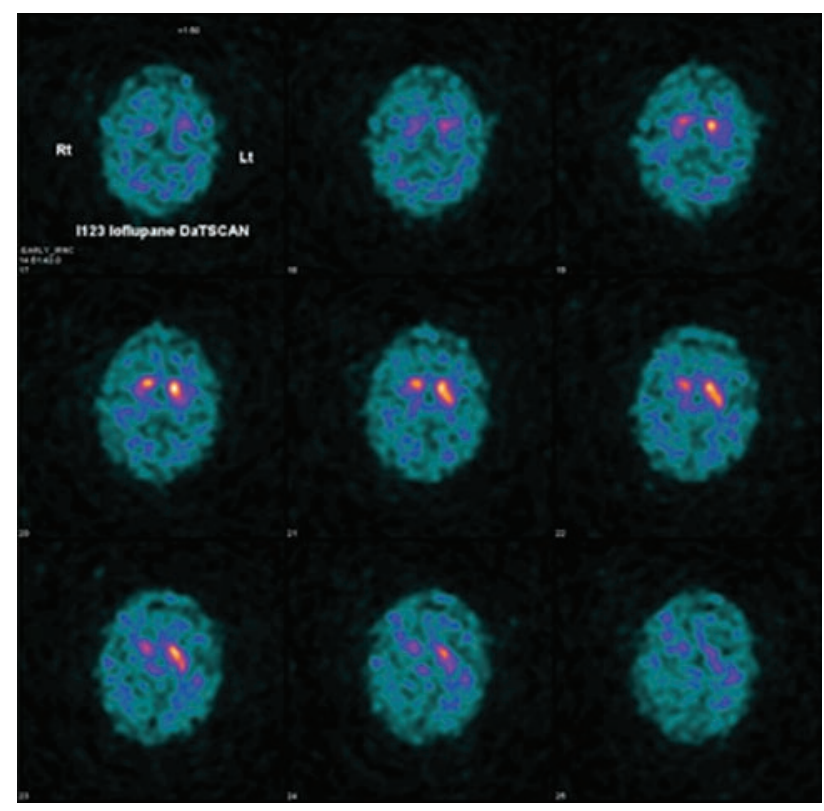

Fig 1. Dopamine transporter scan showing reduced uptake of ioflupane in the right striatum.

\section{Initial management}

The patient required sedation at multiple times during her inpatient stay including benzodiazepines and haloperidol to facilitate tests and care. She was noted to have developed some extrapyramidal signs during the course of her hospital stay. Haloperidol was switched to risperidone. With the non-specific EEG findings a course of intravenous methylprednisolone was trialled for probable autoimmune encephalitis with no effect.

\section{Case progression and outcome}

During the course of her admission, she was noted to have bilateral cogwheel rigidity and bradykinesia albeit these ensued while already on haloperidol and later risperidone suggestive of marked intolerance to these medications. During this period, she required assistance with all her care needs. She suffered occasional urinary incontinence and visual hallucinations of dogs and butchers. She also suffered a fall. Haloperidol, then risperidone was stopped with marked but incomplete resolution of her symptoms. She regained her mobility, became continent albeit continued to exhibit behavioural disturbances including restlessness, paranoia, mood swings and some agitation.

Management with rivastigmine (cholinesterase inhibitor) was initiated once the DaTSCAN result confirmed reduced uptake of ioflupane in the right striatum. She became settled enough for out-of-hospital care although was discharged to a dementia registered home for continuing care.

\section{Discussion}

Acute presentation of dementia is uncommon. Dementia typically develops over months to years allowing a structured holistic approach often in the community with input from specialist memory clinics. In stark contrast are rapidly progressive dementias. These must be investigated without delay and often in a hospital setting as reversible treatment and management are essential to mitigating disease progression and associated mortality. ${ }^{1}$

The initial screening tools available for assessing cognition and delirium are shown in Table 1. These include the AMT-4, confusion assessment method (CAM), AMT-10 and the 4 As test (4AT). Our trust recommends cognitive screening for patients using the AMT- 4 as it is easy and quick to administer. If the AMT-4 score is less than 4 this should be followed up with the CAM or 4AT screening tools for delirium. We would recommend using more comprehensive cognitive assessment tools such as the Montreal cognitive assessment and the Addenbrooke's cognitive assessment (ACE-III) if required in cases where there is suspicion of chronic cognitive dysfunction (dementia).

We suggest a methodical approach in tackling the differential diagnosis. This includes a careful history for any precipitating factors including medications, psychiatric symptoms and infection. This should be followed by investigations for infectious, haematological, metabolic, endocrine, autoimmune, malignant and vascular causes as illustrated in this patient.

Dementia with Lewy bodies (DLB) is an important diagnosis to identify and treat early. The fourth report of the Lewy Body Dementia Consortium of 2017 provides clinicians with core and supportive clinical features in addition to indicative and supportive biomarkers. ${ }^{2}$ Fluctuating cognition, highly detailed visual hallucinations, rapid eye movement sleep disorder and at least one cardinal sign of Parkinsonism (bradykinesia, rigidity and tremor) make the core clinical features (Table 2). Notable in this supportive clinical feature classification is the severe sensitivity to antipsychotics. This has a high sensitivity and specificity for DLB but has been classed as such due to reduced use of this medication. ${ }^{3,4}$

Misdiagnosis of DLB as Creutzfeldt-Jakob disease (CJD) is also common as seen in a large French pathological study ${ }^{5}$ and up to $9 \%$ in a large German study. ${ }^{6}$ Periodic sharp waves (pseudo periodic triphasic complexes) may be present as the disease progresses on EEG and can also contribute to diagnosis of prion disease-CJD, albeit these are seen in progressive disease.

Fluid-attenuated inversion recovery and DWI MRI can however be useful in distinguishing these two pathologies.

EEG can also be of use in differentiating causes of delirium and dementia. $^{7}$

The DaTSCAN is a type of single-photon-emission CT used specifically in the assessment of suspected Parkinson's disease or dementia with Lewy bodies and enables the visualisation of dopaminergic activity in the basal ganglia. It can be used to differentiate DLB from other forms of dementia. The National Institute for Health and Care Excellence recommends that functional neuroimaging with DaTSCAN should be used to help establish diagnosis in those with suspected DLB if the diagnosis is in doubt. ${ }^{8}$

In summary a structured approach ruling out reversible causes of delirium is appropriate but should not limit further investigation for rapidly progressive dementia in selected cases (Table 3).

Another important aspect of care revolves around capacity and deprivation of liberty as in our patient who was restless on occasions requiring physical and chemical restraints.

It is also important to continue to monitor patients and their response to treatment. 
Table 1. Initial screening tools available for assessing cognition and delirium

\begin{tabular}{|c|c|c|c|c|c|c|c|}
\hline $\begin{array}{l}\text { Confusion assessment } \\
\text { method (CAM) }\end{array}$ & \multicolumn{2}{|c|}{$\begin{array}{l}\text { Abbreviated mental } \\
\text { test-4 (AMT-4) }\end{array}$} & \multicolumn{2}{|l|}{$\begin{array}{l}\text { Abbreviated mental } \\
\text { test-10 (AMT-10) }\end{array}$} & \multicolumn{3}{|l|}{4 As test (4AT) } \\
\hline $\begin{array}{l}\text { Acute onset and } \\
\text { fluctuating course }\end{array}$ & Age & 1 & Age & 1 & Alertness & $\begin{array}{l}\text { Normal, mild sleepiness } \\
\text { for }<10 \text { seconds on } \\
\text { walking }\end{array}$ & 0 \\
\hline Inattention & $\begin{array}{l}\text { Date of } \\
\text { birth }\end{array}$ & 1 & Time & 1 & & Clearly abnormal & 4 \\
\hline Disorganised thinking & Place & 1 & Address to recall & 1 & AMT-4 & No mistakes & 0 \\
\hline \multirow{7}{*}{$\begin{array}{l}\text { Altered level of } \\
\text { consciousness }\end{array}$} & Year & 1 & Year & 1 & & 1 mistake & 1 \\
\hline & & & Location & 1 & & $\begin{array}{l}\geq 2 \text { mistakes or } \\
\text { untestable }\end{array}$ & 2 \\
\hline & & & $\begin{array}{l}\text { Recognition of two } \\
\text { persons }\end{array}$ & 1 & $\begin{array}{l}\text { Attention, listing } \\
\text { months in reverse order }\end{array}$ & $\geq 7$ months correctly & 0 \\
\hline & & & Date of birth & 1 & & $\begin{array}{l}<7 \text { months correct or } \\
\text { refuses to start }\end{array}$ & 1 \\
\hline & & & $\begin{array}{l}\text { Year that World War } \\
\text { II started }\end{array}$ & 1 & & Untestable & 2 \\
\hline & & & Present monarch & 1 & $\begin{array}{l}\text { Acute change or } \\
\text { fluctuating course }\end{array}$ & No & 0 \\
\hline & & & $\begin{array}{l}\text { Counting backwards } \\
\text { from } 20-1\end{array}$ & 1 & & Yes & 4 \\
\hline
\end{tabular}

\section{Key learning points}

$>$ Consider a wide range of differentials when elderly patients present with acute confusion.
> The early diagnosis of Lewy body dementia is clinically important in terms of avoidance of neuroleptic agents and appropriate pharmacological management with the use of cholinesterase inhibitors especially rivastigmine.

Table 2. Diagnosis of dementia with Lewy bodies. Probable dementia with Lewy bodies can be diagnosed with $\geq 2$ core clinical features, or one core clinical feature and $\geq 1$ indicative biomarker. Possible dementia with Lewy bodies can be diagnosed with only one core clinical feature or $\geq 1$ indicative biomarker without a core clinical feature. Adapted with permission from McKeith IG, Boeve BF, Dickson DW et al. Diagnosis and management of dementia with Lewy bodies: Fourth consensus report of the DLB Consortium. Neurology 2017:89:88-100.

\section{Core clinical features \\ Fluctuating cognition \\ Recurrent visual hallucinations \\ REM sleep behaviour disorder \\ $\geq 1$ cardinal feature of Parkinsonism}

\section{Indicative biomarkers}

Reduced dopamine transporter uptake in basal ganglia (by SPECT or PET) Abnormal ${ }^{123}$ iodine-MIBG myocardial scintigraphy (low uptake)

REM sleep without atonia (polysomnographic confirmation)

\section{Supportive clinical features}

Severe sensitivity to antipsychotics

Non-visual hallucinations

Hypersomnia, hyposmia, delusions, apathy, anxiety, depression

Postural instability

Episodes of unresponsiveness

\section{Supportive biomarkers}

Relative preservation of medial temporal lobe (by CT/MRI) Generalised low uptake perfusion/metabolism scan with reduced occipital activity (by SPECT/PET)+/- the cingulate island sign (by FDG-PET)

Prominent posterior slow-wave activity with periodic fluctuations in the pre-alpha/theta range (by EEG) 
Table 3. Approach to investigating acute and or chronic cognitive dysfunction

\begin{tabular}{|c|c|c|}
\hline Modality & Test & Conditions \\
\hline \multirow[t]{8}{*}{ Bloods } & $\begin{array}{l}\text { Full blood count, urea and electrolytes, calcium, B12, folate, } \\
\text { thyroid function tests, cortisol, liver function tests, ammonia, } \\
\text { HIV, microscopy and culture, arterial blood gas, glucose }\end{array}$ & \multirow{8}{*}{$\begin{array}{l}\text { Infection, anaemia, electrolyte } \\
\text { imbalance, dehydration, liver failure, } \\
\text { renal failure, endocrinopathy, vitamin } \\
\text { deficiency, septicaemia, hypoxia, } \\
\text { hypercapnia, acid base disorders, } \\
\text { glycaemic disorders, autoimmune } \\
\text { encephalitis }\end{array}$} \\
\hline & Anti-thyroid peroxidase antibody, thyroid-stimulating hormone & \\
\hline & Anti-nuclear IgG antibodies & \\
\hline & $\begin{array}{l}\text { dsDNA, Sm/RNP, Ro52, Ro60, La (SS-B), Scl-70, centromere, } \\
\text { Mi-2, Ku, Th/To, RNA Pol III, Pm-Scl and proliferating cell nuclear } \\
\text { antigen, Jo-1 and ribosomal-P }\end{array}$ & \\
\hline & Anti-neutrophil cytoplasmic IgG antibodies screen & \\
\hline & Anti-glutamic acid decarboxylase IgG antibody & \\
\hline & $\begin{array}{l}\text { Anti-nuclear IgG antibodies by indirect immunofluorescence } \\
\text { (HEp-paraneoplastic IgG antibody screen) }\end{array}$ & \\
\hline & Voltage gated potassium channel antibodies & \\
\hline Urine & Urine analysis, microscopy and culture, toxicology screen & Urinary tract infection, drug toxicosis \\
\hline Ultrasound & Bladder, kidney-ureter-bladder, echocardiography & $\begin{array}{l}\text { Urinary retention, urinary tract infection, } \\
\text { subacute bacterial endocarditis }\end{array}$ \\
\hline X-ray & Chest, musculoskeletal & Pneumonia, heart failure, fracture \\
\hline Neuroimaging & CT head, MRI head, DaTSCAN & $\begin{array}{l}\text { Stroke, haemorrhage, structural lesions, } \\
\text { dementia with Lewy bodies }\end{array}$ \\
\hline Computed tomography & Chest, abdomen and pelvis & Suspecting malignancy \\
\hline $\begin{array}{l}\text { Magnetic resonance } \\
\text { imaging }\end{array}$ & DWI, FLAIR & SOL, Alzheimer's disease, CVA \\
\hline \multirow[t]{8}{*}{ Lumbar puncture } & CSF protein & \multirow[t]{8}{*}{ Meningitis, encephalitis } \\
\hline & CSF glucose & \\
\hline & CSF TPO, M/C/S & \\
\hline & Oligoclonal bands & \\
\hline & NMDA receptor antibodies & \\
\hline & Voltage gated calcium channel antibodies & \\
\hline & Voltage gated potassium channel antibodies & \\
\hline & CSF (s100, p 14-3-3) in CJD & \\
\hline Electroencephalography & $\begin{array}{l}\text { Pseudo periodic triphasic complexes may be present progressive } \\
\text { disease in prion disease (CJD) }\end{array}$ & $\begin{array}{l}\text { Delirium, metabolic eg hepatic } \\
\text { encephalopathy, epilepsy }\end{array}$ \\
\hline DaTSCAN & Reduced ioflupane uptake & Dementia with Lewy bodies \\
\hline
\end{tabular}

\section{References}

1 Geschwind MD, Haman A, Miller BL. Rapidly progressive dementia. Neurol Clin 2007:25:783-807.

2 McKeith IG, Boeve BF, Dickson DW et al. Diagnosis and management of dementia with Lewy bodies: Fourth consensus report of the DLB Consortium. Neurology 2017:89:88-100.

3 Baskys A. Lewy body dementia: the litmus test for neuroleptic sensitivity and extrapyramidal symptoms. J Clin Psychiatry 2004;65(Suppl 11):16-22.

4 McKeith I, Fairbairn A, Perry R, Thompson P, Perry E. Neuroleptic sensitivity in patients with senile dementia of Lewy body type. BMJ 1992:305:673-8.

5 Haik S, Brandel JP, Sazdovitch V et al. Dementia with Lewy bodies in a neuropathologic series of suspected Creutzfeldt-Jakob disease. Neurology 2000;55:1401-4.
6 Heinemann U, Krasnianski A, Meissner B et al. Creutzfeldt-Jakob disease in Germany: a prospective 12-year surveillance. Brain 2007:130:1350-9.

7 van der Zande JJ, Gouw AA, van Steenoven I et al. EEG Characteristics of dementia with lewy bodies, alzheimer's disease and mixed pathology. Front Aging Neurosci 2018;10:190.

8 National Institute for Health and Care Excellence. Dementia: assessment, management and support for people living with dementia and their carers. NICE guideline [NG97]. NICE, 2018.

Address for correspondence: Dr Olugbenro Akintade, Peterborough City Hospital, Edith Cavell Campus, Bretton Gate, Peterborough, Cambridgeshire PE3 9GZ, UK. Email: olugbenro.akintade@nhs.net 